\title{
Work-related factors and depressive symptoms in firefighters - preliminary data
}

\author{
Magdalena Konopko ${ }^{1}$, Wojciech Jarosz ${ }^{2}$, Przemysław Bienkowski ${ }^{3}$, Halina Sienkiewicz- \\ Jarosz ${ }^{1, *}$ \\ ${ }^{1}$ Institute of Psychiatry and Neurology, 1st Department of Neurology, 9 Sobieskiego St. 02-957 \\ Warsaw, Poland \\ ${ }^{2}$ The Main School of Fire Service, Faculty of Fire Safety Engineering, 52/54 Slowackiego St., 01-629 \\ Warsaw, Poland \\ ${ }^{3}$ Medical University of Warsaw, Department of Psychiatry, Faculty of Health Sciences, 61 Żwirki i \\ Wigury St., 02-091 Warsaw Poland
}

\begin{abstract}
Introduction: Firefighters participate in rescue activities with intense physical and psychological stress. The aim of the present study was to assess prevalence of depression and work-related stress in firefighters. Methods and findings: A questionnaire including items related to sociodemographic, health-related and work-related issues has been used. Depressive symptoms were assessed with PHQ-9 scale. Data were collected from 72 fire service workers ( 9 females). The mean age was $30.1 \pm 7.5$ and $46.0 \pm 8.6$ years for men and women respectively. $86.1 \%$ declared no chronic disease, $5.5 \%$ - hypertension and $4.2 \%$ endocrine diseases. $62.5 \%$ were never smokers and $19.4 \%$ current smokers. $25 \%$ of the group declared drinking alcohol more than 2 times a week. More than $33.0 \%$ declared significant work-related stress. The main reasons were: lack of time to perform all activities $(27.0 \%)$, low influence on work performance $(15.3 \%)$, low pay $(33 \%)$ and unequal treatment in the workplace $(79.0 \%)$. Depressive symptoms were reported by $23 \%$. Ten percent reported burnout symptoms and stress related to work. Conclusion: The occupational obligations and work-related stress may be responsible for the psychological problems experienced by firefighters.
\end{abstract}

\section{Introduction}

Depression and depressive symptoms are one of the potential factors that can affect the quality of life, the ability to work and work effectiveness. So far, there is paucity of data on how and why people cease their professional work due to depressive disorders in Poland. It is well known, however, that depression is at the top of the main causes of long-term sickness absence [1]. Undoubtedly, depression has a negative impact on the overall health, because it worsens compliance or even is a cause of lack of adequate prevention and treatment of somatic diseases, so, it seems important to study potential causes of depressive symptoms in workers. To date, some studies have evaluated the role of socioeconomic and

\footnotetext{
*Corresponding author : jarosz@ipin.edu.pl
} 
demographic factors on depression [2, 3]. Another study performed on general population in Brazil had shown, that personal and occupational factors can be also related to depression [4]. The main occupational risk factors were: part time working ( $\leq 20 \mathrm{~h}$ a week) and stress at work, workplace violence, intense physical activity, exposure to noise and chemicals among women, and prolonged exposure to sun among men [4].

Firefighters are exposed to various harmful factors, such as urgent calls and an irregular daily schedule. This kind of occupational environment can lead to various health problems such as physical and mental distress. Anxiety and depression, abuse risk and burnout symptoms can be more prevalent in this population [5-8]. A recent study has reported that about $5 \%$ of firefighters suffer from depression and $8 \%$ from post-traumatic stress disorder [9]. In Fullerton et al. study [10], prevalence of depression was even higher and reached $22 \%$. These health problems may be thought of as a personal problem but should also be considered a social issue because firefighters serve as government officers in charge of social wellbeing.

The aim of the present preliminary study was to assess prevalence of depression and work-related stress in firefighters and to assess their relationship with general health, sociodemographic and work-related variables.

\section{Methods}

We have developed a tool dedicated for detecting depressive symptoms and assessing variables probably having impact on their development in professionally active people. Questionnaire consisted of several parts: (I) sociodemographic data, (II) PHQ-9 questionnaire, which is one of the most commonly used tools for diagnosis of depression $[11,12]$, (III) questions regarding potential symptoms including the bipolar spectrum based on hypomania checklist $[13,14]$, (IV) questions characterizing the work performed and questions indicating the planned cessation of the current work. The layout of the questionnaire and its individual items have been prepared in a way that allows to assess the relationship between the severity of depressive symptoms (part II, PHQ-9), bipolar spectrum (part III) and demographic, clinical and socio-economic conditions (part I) and conditions related to with professional work (part IV).

The study has been approved by the Ethics Committee of the Institute of Psychiatry and Neurology. All responders gave informed consent for participation in the study.

The results are presented as mean \pm standard deviation. The degree of association between variables was determined using Pearson's coefficient (r). All statistical analysis has been performed using Statistica (Statsoft, version 13") and $\mathrm{p}<0.05$ have been considered as statistically significant.

\section{Results}

We present a preliminary data collected from 72 professionally active fire service workers ( 63 males and 9 females). The mean age of participants was $30.1 \pm 7.5$ and $46.0 \pm 8.6$ years for men and women respectively and the mean job duration of firefighting experience was $10.0 \pm 11.6 .51 .0 \%$ of participants have been married. Majority $(61.1 \%)$ lived in villages or towns smaller than 50.000 of inhabitants. More than thirty percent declared university education.

The respondents were generally healthy $-86.1 \%$ declared no chronic disease. The most frequently reported were hypertension $-5.5 \%$ and endocrine diseases $-4.2 \%$. 62.5\% were never smokers and $19.4 \%$ and $16.6 \%$ were current smokers or past smokers, respectively. Twenty five percent declared drinking alcohol more than 2-3 times a week and 50.0\% 2-4 
times a month. Two of respondents declared treatment of alcohol abuse, 3 - current depression treatment and 7 - family history of depression.

Family relationships have been reported as very good by $34.7 \%$ and by $62.5 \%$ as good, with very rare conflicts with other family members. Feeling of loneliness, which can predispose to depression, have been reported by only $3.0 \%$ of the study group, $13.9 \%$ reported, that they feel so "sometimes" and $40.3 \%$ that it happens rather "rarely".

Eighty one percent of the study group assessed their economic status as good or very good. 59.7\% of the group declared lack of important stressful life events during past year, $13.9 \%$ reported serious partner's disease, $11.1 \%$ death of family member and $5.0 \%$ suffered from severe disease.

More than $33.0 \%$ of firefighters declared significant work-related stress. The main reasons were: lack of time to perform all activities (27.0\%), low influence on work performance $(15.3 \%)$, low pay $(33.0 \%)$ and unequal treatment in the workplace $(79.0 \%)$. $93 \%$ of respondents work on permanent contract.

Depressive symptoms have been present in about $23 \%$ (18.5\% have mild symptoms, $4.2 \%$ moderate and $1.4 \%$ severe) and bipolar spectrum symptoms in about $15 \%$. More than one tenth of the group reported stress related to work: with psychosomatic problems in $11.1 \%$, insomnia in $36.1 \%$, and fear of going to work in $12.5 \% .8 .3 \%$ reported thoughts about profession change. Physical and mental work ability have been generally highly assessed as very good or good in more than $90.0 \%$ of the study group, and majority of respondents declared ability to work during approaching years. Only $8.5 \%$ declared more than 10 days of sickness absence during the last 12 months.

Age, job duration, working hours a week are similar in subgroups with different depression symptoms severity.

Significant correlations have been found between feeling of loneliness and depressive symptoms $(\mathrm{r}=0.32 ; \mathrm{p}=0.007)$, between severity of depression and fear of going to work $(\mathrm{r}=0.35 ; \mathrm{p}=0.002)$, and between depression severity and problems with sleep $(\mathrm{r}=045$; $\mathrm{p}=0.0002)$. No correlations between depressive symptoms severity, health and socioeconomic status were found.

\section{Discussion}

The study aimed: (i) to assess the prevalence of depression and work-related stress in firefighters and(ii) to assess their relationship with general health, sociodemographic and work-related variables.

The results revealed high rates of depressive symptoms of different severity among participants $(23 \%)$, although moderate or severe depression has regarded $5.6 \%$. Such prevalence of moderate and severe depression has been lower than observed in the previous studies $[15,16]$. One explanation can be different scale used for detection of depressive symptoms (Beck Depression Inventory and Centre for Epidemiologic Studies Depression Scale vs. PHQ-9), different age of the studied cohorts, and possibly other work organisation systems, and different socioeconomical aspects. On the other hand, the prevalence of depressive symptoms in Polish firefighters is like that observed in urban population of Poland [17].

In another study the most frequent problems found in firefighters were burnout symptoms (20\%) and symptoms consistent with post-traumatic stress disorder (PTSD) [18]. In the present study, we have addressed the question of potential causes and symptoms of work burnout. Stress related to work inducing psychosomatic problems has been reported by $11.1 \%$, and was lower than reported previously $[18,19]$. The insomnia has been reported by $36.1 \%$ of fire service workers, and this number is lower compared to $52.7 \%$ in the Hom et al study [16] and near $60 \%$ in Carey et al. study [15]. $12.5 \%$ of study participants 
reported fear of going to work and $8.3 \%$ reported thoughts about changing the profession. It is rather worrisome trend, which probably need more attention and development of preventive strategies. We didn't find important differences between groups with depressive symptoms of different severity and age, job duration, and working hours a week.

We found significant correlations between feeling of loneliness and depressive symptoms $(\mathrm{r}=0.32 ; \mathrm{p}=0.007)$. This observation is not surprising, because loneliness has been associated with negative mental health outcomes such as depression, suicidality, poor sleep quality and general health. It has been also considered a major source of psychological stress [20].

The correlation between depression severity and problems with sleep has supported the role of insomnia as a cardinal symptom of depression, and probably potential precursor or even prodromal feature of depression [16]. The relationship between sleep problems and depression in firefighters has been reported in previous studies $[15,16]$. The link between insomnia and suicidal ideation and behaviours has been also suggested [21]. In the present study, depressive symptoms correlated with fear of going to work $(r=0.35 ; \mathrm{p}=0.002)$, which may suggest the role of work-related stress in development of depression [4]. No correlations between depressive symptoms severity, health and socioeconomic status were found.

The limitation of the present study is rather small study group and one-point study without longitudinal assessment of reported problems, especially related to work environment.

\section{Conclusion}

That preliminary data has shown that prevalence of depression in Polish firefighters does not exceed that reported in another studies. The occupational obligations and work-related stress may be one of the causes of psychological problems experienced by Polish firefighters. Depressive symptoms in this group are possibly associated with other psychological and medical problems, including feeling of loneliness and sleep disturbances. Further studies with larger samples are needed to uncover causal associations between work related stress and depressive symptoms in firefighters.

Publication based on the results of the fourth stage of the multi-annual program "Improving safety and working conditions" financed in 2017-2019 in the field of research and development from the funds of the Ministry of Science and Higher Education/National Centre for Research and Development. Program coordinator: Central Institute for Labour Protection - National Research Institute (PBiWP-IV/2017 Sub-contract No. TP-48/2017/PW-PB project I.N.08).

\section{References}

1. ZUS Statistics (2016)

2. V. Lorant, C. Croux, S. Weich, D. Deliege, J. Mackenbach, M. Ansseau, Brit. J. Psychiat. 190 (2007)

3. V. Patal, R. Araya, M. de Lima, A. Ludermir, C. Todd, Soc. Sci. Med. 49 (1999)

4. N.S.X. Oenning, P.K. Ziegelmann, B.N.G. Goulart, I. Niedhammer, J. Affect. Disord. 240 (2018)

5. M.L. Pennington, T.P. Carpenter, S.J. Synett, V.A. Torres, J. Teague, S.B. Morissette, J. Knight, B. W. Kamholz, T. M. Keane, R. T. Zimering, S. B. Gulliver, Prehosp. Disaster Med. 33 (2018) 
6. J.W. Boffa, I.H. Stanley, L.J. Smith, B.M. Mathes, J.K. Tran, S.J. Buser, N.B. Schmidt, A.A. Vujanovic, J .Nerv. Ment. Dis. 206 (2018)

7. J.I. Kim, H. Park, J.H. Kim, J. Affect. Disord. 15 (2018)

8. C. Psarros, C. Theleritis, N. Kokras, D. Lyrakos, A. Koborozos, O. Kakabakou, G. Tzanoulinos, P. Katsiki, J.D. Bergiannaki, Nord. J. Psychiatry 72 (2018)

9. S.B. Harvey, J.S. Milligan-Saville, H.M. Paterson, E.L. Harkness, A.M. Marsh, M. Dobson, R. Kemp, R.A. Bryant, N.Z. Aust, J. Psychiatry 50 (2016).

10. C.S. Fullerton, R.J. Ursano, L. Wang, Am. J. Psychiatry 161 (2004)

11. K. Kroenke, R.L. Spitzer, J.B. Williams, J. Gen. Intern. Med. 16 (2001)

12. L. Manea, S. Gilbody, D. McMillan, CMAJ 184 (2012)

13. D. Łojko, D. Dudek, J. Angst, M. Siwek, M. Michalak, J. Rybakowski, Psychiatr Pol. 50 (2016)

14. Ł. Święcicki. Materiały edukacyjne (2011)

15. M.G. Carey, S.S. Al-Zaiti, G.E. Dean, L. Sessanna, D.S. Finnell, J. Occup. Environ. Med. 53 (2011)

16. M.A. Hom, I.H. Stanley, M.L. Rogers, M. Tzoneva, R.A. Bernert, T.E. Joiner, J. Clin. Sleep Med. 12 (2016)

17. S. Bell, A. Britton, R. Kubinova, S. Malyutina, A. Pajak, Y. Nikitin, M. Bobak, PLoS One. 13, 9 (2014)

18. S. Mitani, M. Fujita, K. Nakata, T Shirakawa T.J Emerg, Med. 31 (2006)

19. M. Kaflik-Pieróg, N. Ogińska-Bulik, Acta Univ Lodz. 7 (2003)

20. M.E. Beutel, E.M. Klein, E. Brähler, I. Reiner, C. Jünger, M. Michal, J. Wiltink, P.S. Wild, T. Münzel, K.J. Lackner, A.N. Tibubos, BMC Psychiatry 20 (2017)

21. R.A. Bernert, J.S. Kim, N.G. Iwata, M.L. Perlis, Curr. Psychiatry Rep. 17 (2015) 